\title{
The FARCOS project - Status and perspective
}

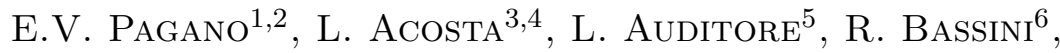 \\ C. Boiano ${ }^{6}$, G. CARdella $^{3}$, A. Castoldi ${ }^{6,7}$, M. D'Andrea ${ }^{3}$,
} E. De Filippo ${ }^{3}$, F. Fichera ${ }^{3}$, L. Francalanza ${ }^{1,2}$, N. Giudice $^{3}$,

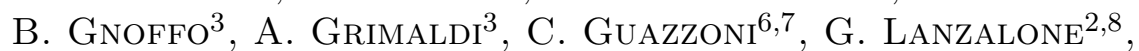
I. Lombardo ${ }^{9}$, T. Minniti ${ }^{1,3}$, E. Morgana ${ }^{5}$, A. Pagano $^{3}$, M. PAPA $^{3}$, G. Passaro ${ }^{2}$, S. Pirrone ${ }^{3}$, G. Politi ${ }^{1,3}$, F. Porto ${ }^{1,2}$, L. Quattrocchi ${ }^{5}$, F. Rizzo ${ }^{1,2}$, E. Rosato ${ }^{9}$, P. Russotto ${ }^{3}$, G. SACCÀ ${ }^{3}$, A. Trifirò ${ }^{5}$, M. Trimarchi ${ }^{5}$, G. Verde $^{3}$ and M. VigiLANTE ${ }^{9}$

${ }^{1}$ Dip. di Fisica e Astronomia, Università di Catania, Catania, Italy

${ }^{2}$ INFN-Laboratori Nazionali del Sud, Catania, Italy

${ }^{3}$ INFN, Sezione di Catania, Catania, Italy

${ }^{4}$ Departamento de Fisica Aplicada, Universidad de Huelva, Huelva, Spain

${ }^{5}$ INFN, Gruppo Collegato di Messina and Dipartimento di Fisica e

Scienze della Terra, Università di Messina, Messina, Italy

${ }^{6}$ INFN, Sezione di Milano, Milano, Italy

7 Politecnico di Milano, Milano, Italy

${ }^{8}$ Kore Università di Enna, Enna, Italy

${ }^{9}$ INFN, Sezione di Napoli and Dip. di Fisica, Univ. di Napoli, Italy

\begin{abstract}
Nuclear matter under extreme conditions can be studied in the laboratory with heavy-ion collisions. In this case one can indeed vary the incident energy and explore different impact parameter and rapidity regions to access densities that extend above and below saturation. This opportunity allows one to learn about the nuclear equation of state and its implications in astrophysics phenomena such as supernovae explosions and neutron stars properties. All these phenomena involve time
\end{abstract}


scales that need to be accessed with two- and multi-particle correlation measurements. In this contribution a brief report of the FARCOS array will be presented. In particular, tests with alpha sources and beams and preliminary results from a recent experiment INKIISSY performed using a FARCOS prototype made of four telescopes will be presented.

\section{Introduction}

The study of dynamics in heavy ion collisions (HIC) is an active field in nuclear physics. In fact, depending on incident energy and impact parameter, one is faced with different time-scales and scenarios of the response of the nucleus to the excitation of the relevant degrees of freedom. This opens the possibility to explore transient systems with baryonic density lower or larger than the one characterizing the saturation properties of normal nuclear matter $\left(0.17 \mathrm{nuc} / \mathrm{fm}^{-3}\right)$. At intermediate energies between $20 \mathrm{MeV} / \mathrm{u}$ and $200 \mathrm{MeV} / \mathrm{u}$, Fermi energies, within time-scales of the order of $100 \mathrm{fm} / \mathrm{c}$, violent head-on collisions experience a collective compression where the nuclear density in the overlapping region is predicted to achieve values well above the value of the saturation density $[1,2]$. Then, the evolution of the system is predicted to undergo an expansion phase: the density degreases to a freeze-out configuration with significant lower values (of the order of $0.3 \rho_{0}$ ) and a multi-fragmentation of the system in many excited clusters and light particles takes place. It is still a fundamental open question if at freezeout the system achieves full equilibrium or not. Energetic HIC have been extensively studied to extract information about the properties of nuclear matter under extreme conditions [3]. These studies have shown that besides nuclei lying close to the stability valley also exotic isotopes are produced in the dynamical phase of the reaction. In particular, the decay properties of the exotic such isotopes of short life-decay time can be studied by measuring the correlation properties of the emitted particles in order to probe valuable spectroscopic information about unbound exotic states that can be isolated by means of this techniques [4-10]. From this point of view HIC can be viewed not only as a powerful tool to study nuclear dynamics and the evolutive path towards equilibrium configurations but also as a unique terrestrial laboratory to produce several nuclear unbound species in one single experiment and to study same of their spectroscopic properties. With the advent of new-generation radioactive ion-beam facilities, such as FAIR at GSI , FRIB at MSU, SPES at LNL or SPIRAL II at GANIL, these studies and methods assume renewed interest in order to study both the structure of 


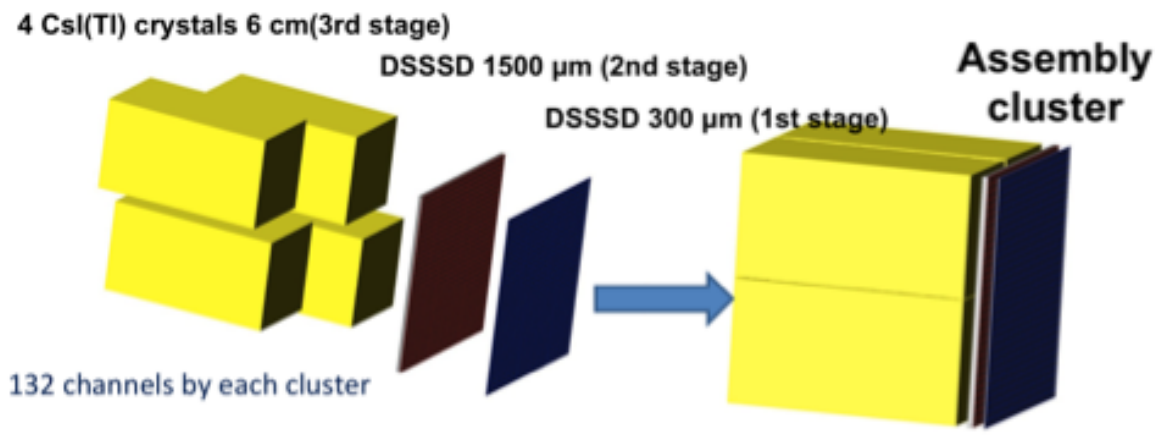

Figure 1: Schematic view of a cluster of FARCOS.

rare isotopes and the propety of isospin asymmetric nuclear matter with interesting implications in nuclear astrophysics. From the experimental point of view, both high energy and angular resolution of the detection systems are mandatory. For this reason it is necessary to design specific position sensitive detector arrays coupled to $4 \pi$ detectors in order to characterize the reaction event-by-event. In this paper we briefly describe the correlator FARCOS (Femtoscope ARray for COrrelation and Spectroscopy) [11,12] that is under construction at INFN, Sezione di Catania and LNS, in collaboration with INFN Sezione di Milano and Politecnico di Milano. We briefly describe also its first tests with beams at LNS in Catania, performed on April 2013.

\section{FARCOS}

FARCOS is a modular array of telescopes each one consisting of three detection stages. The first two stages are two Double Sided Silicon Strip Detectors (DSSSD) with 32 vertical strips in the front side and 32 horizontal strips in the back side. For both the area is $6.4 \times 6.4 \mathrm{~cm}^{2}$ and the first silicon detector is $300 \mu \mathrm{m}$ thick and the second one is $1500 \mu \mathrm{m}$ thick. The last stage consists of four $\mathrm{CsI}(\mathrm{Tl})$ crystals of $6 \mathrm{~cm}$ of thickness read by photodiodes, with an active area of $2 \times 2 \mathrm{~cm}^{2}$ Fig. 1 . shows the three stages of a single cluster of FARCOS (left side) and their typical close assembling (right side). It is important to note that each cluster is equipped with 132 independent read-out electronics channels (128 for silicon strips and 4 for CsI photodiode readout). 
Particle Discrimination techniques in FARCOS are performed by means of popular $\triangle \mathrm{E}$-E technique. The available electronics allows also pulse shape(PSD) and Time of Light (TOF) methods. Beside the compact design, portability and modularity are important features making it easy to couple the array to $4 \pi$ detectors, other correlators and magnetic spectrometers. For the preliminary prototypes, we have used modified versions of CHIMERA-like standard preamplifiers (PAC) for both $\mathrm{CsI}(\mathrm{Tl})$ crystals and silicon detectors already developed to match with the necessity of low electronics noise and TOF performance [13]. Starting from these studies, for the reading of the silicon strips we are testing new pseudo-differential 32Ch PACs-low power consumption ( $<900 \mathrm{~mW}$ for 32 channels) that are very compact (86x80x10 mm (NPA-16FL), 98x80x15 mm (NPA-16FE) ) [14].

\subsection{Characterization of FARCOS with Beam, Identifications and calibrations procedures}

First tests of FARCOS prototype was devoted to characterize the surface light response and the bulk properties of the $\mathrm{CsI}(\mathrm{Tl})$ crystals [15]. It was found a good uniformity both in the surface light response and depth inside the crystals about $1.5 \mathrm{~cm}$ [16]. FARCOS was tested and coupled with the $4 \pi$ CHIMERA for the first time during the experiment INKIISSY (INverse KInematic ISobaric System) on April 2013, performed at INFN-LNS of Catania using the K800 Superconducting Cyclotron. The idea of this experiment was to use projectile/target combination having the same mass of the neutron rich ${ }^{124} \mathrm{Sn}+{ }^{64} \mathrm{Ni}$ system and $\mathrm{N} / \mathrm{Z}$ similar to the neutron poor ${ }^{112} \mathrm{Sn}+{ }^{58} \mathrm{Ni}$ one, that is ${ }^{124} \mathrm{Xe}+{ }^{64} \mathrm{Zn}$, at the same bombarding energy of 35 $\mathrm{MeV} / \mathrm{u}$ as in previous experiments [17], in order to disentangle Isospin from size effects in dynamical fission the projectile-like fragment. The setup consisted of the $4 \pi$ detector CHIMERA and 4 modules of FARCOS prototype. In the experimental configuration FARCOS covered a theta angle in the lab frame in the range of polar angles $\theta_{l a b}=16^{\circ}-44^{\circ}$ and azimuthal opening of value $\Delta \phi=75^{\circ}$. The detector was located at a distance of $25 \mathrm{~cm}$ far from the target. After a cut in the total strips hits multiplicity (aimed at removing unphysical events) $\left(1 \leq\right.$ mult $\left._{D S S S D} \leq 8\right)$ good $\triangle \mathrm{E}$-E identification matrixes between the first two DSSSD have been obtained.

For the identification of the particles and for energy and time calibrations is highly recommended to adopt automatic procedures in order to optimize the time needed for calibrations. However, in order to achieve a satisfactory method for saving of time and man power it is necessary to study in detail the response function of independent strips or a suitable ensemble of them. 
It is possible to choose two simple ways to perform the identification. One way consist of studying the identification $\Delta \mathrm{E}-\mathrm{E}$ strip by strip. Evidently, due to the high segmentation of the detectors, this procedure would result to be very time consuming. Alternatively, it is possible to sum up group of strips. If, for example, 8 strips are summed up together, the expected effort for data analysis may be substantially reduced by about a factor of 6 by reducing the number of identification matrixes to be analyzed. In principle it should be possible to add statistics of all the strips in the same DSSSD by assuming that the differences in the thickness among the different strips are negligible. However, due to the independent electronic channels for each strip it is necessary to cross-calibrate all the pulses of the electronic signals (from preamplifier to DAQ) or, even better, to perform independent energy calibration for each of two stages of DSSSD. On Fig. $2 \Delta \mathrm{E}$-E identification matrixes and their identification patterns are shown in the two ways as described above.
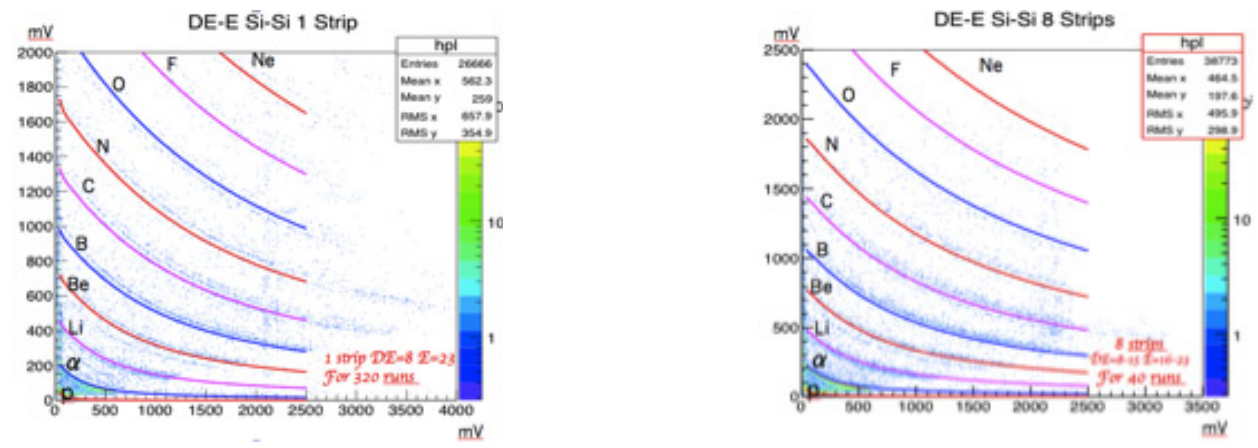

Figure 2: Left panel: $\Delta \mathrm{E}-\mathrm{E}$ for 1 strip in both first and second stages. Right panel: $\Delta \mathrm{E}-\mathrm{E}$ for 8 strips in both first and second stages.

On Fig. 2 (left panel) the $\Delta$ E-E identification matrix for only one strip ( corresponding to the n. 8 in the first DSSSD stage and n. 23 in the second stage) is reported. The statistical sample integrates 320 experimental runs. The typical $\Delta \mathrm{E}-\mathrm{E}$ identification pattern and corresponding fits are also illustrated. In Fig. 2 (right panel), instead, the identification pattern is obtained by adding the statistics of 8 strips (from n. 8th to the $n$. 15th in the first stage and from n. 16th to n. 23th in the second stage), by integrating 40 experimental runs in order to compare with similar significant statistics the two procedures. Notice that for light ions $3 \leq \mathrm{Z} \leq 6$, where $\mathrm{Z}$ is the atomic number, both methods have shown a comparable and satisfactory resolution (see left panel of Fig. 3). In the range of atomic number $7 \leq \mathrm{Z} \leq 8$ the isotopic identification resolution is better in the case of "one-strip way". 
For $9 \leq \mathrm{Z} \leq 10$ in the case of "one-strip way" a good isotopic identification resolution is preserved, but no definitive conclusions were possible for the comparison because there was not enough statistics in the case of 8 added strips in a single matrix (see right panel of Fig. 3).
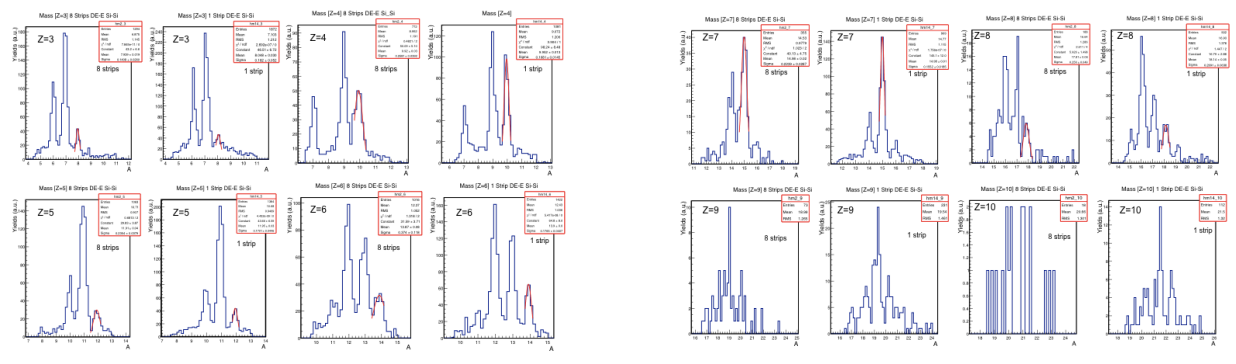

Figure 3: Left panel: Isotopic identification resolution for particle having charge between $3 \leq \mathrm{Z} \leq 6$. Right panel:I sotopic identification resolution for particle having charge between $7 \leq \mathrm{Z} \leq 10$.

In the case of single strip - single-strip identification we observe that: a) wide identification range and good isotopic identification resolution up to atomic number $\mathrm{Z}=10$ (as allowed by collected statistics) have been obtained with no need of energy calibrations or analogic pulse matching. In this procedure we preserved a good quality in spite of the relatively high background due to the small distance between the detector and the target. b) The identification procedure is very time-consuming, using standard procedures. In fact about 100 identification matrixes for each cluster have to be analyzed with high statistics due to the small solid angle covered by each strip $\left(0.2 \times 6.4 \mathrm{~cm}^{2}\right)$. When we analyze the data by summing up groups of strips, we observe that:

c) A good isotopic identification resolution up to atomic number $Z=6-7$ is obtained with optimal resolution for light fragments with $\mathrm{Z}<4$. The procedure is good enough for experiment devoted to light particles and fragments $(\mathrm{Z}<5)$ correlation studies. The method is time-saving. In fact, in the case of the statistics integrated by adding 8 strips together the number of identification matrixes is 16 for each cluster. The method is mandatory if the statistics in strip-by-strip analysis is poor, but, evidently it is necessary an energy calibration (or relative analogic pulse matching) to cross-calibrate the independent electronic channels. 


\section{Conclusions}

Milestones of FARCOS constructions can be briefly summarized as it follows. A new electronic very compact and portable, The GET electronics [20] is under testing in Catania to use it in FARCOS. More details about GET electronics system can be found on the ACTAR and SAMURAI TPC design report, where this electronics is properly used for gas detector $[18,19]$. The test will be completed within the first semester of 2015 year, while in the second semester it is expected to start with procedures to test the ASIC preamplifiers designed by the Politecnico of Milano. In 2016 we will assemble six new clusters; in 2017 and in 2018 we will assemble four new clusters for each year. The final configuration of 20 clusters of FARCOS is expected to be completed on 2019 .

In conclusion, we have performed different tests to extract performances of four clusters of FARCOS correlator. The obtained results are encouraging and indicate both good energy and angular resolutions allowing for satisfactory isotopic identification for light fragments and light charged particles. In the spirit of portability and versatility for both mechanical handling and data analysis (On line and off line), some procedures are under developing to make faster and easier the calibration and the identification analysis. About the electronics front end and the data acquisition, the GET compact integrated electronic, could represent an appropriate and feasible method to process a very large number of channels in a portable and compact way, provided a well designed preamplifier stage is implemented into the system which was not originally designed for silicon and CsI(Tl) detectors. Full tests are in progress. The second part of the 2015 it is envisaged to test new ASICs preamplifiers under development that will be integrated in the mechanics of FARCOS in order to be coupled with GET. In the next five years, it is expected to complete the configuration of 20 clusters of FARCOS. With the new device further contributions will be expected in addressing open problems in nuclear physics and astrophysics and in opening new perspectives. Since 2015 year FARCOS will play an important role in two experiments already approved at LNS, CLIR and SIKO. Beside that, the detector is also involved in a couple of Letters of Intent submitted to the new radioactive Ion facility, SPES, that is under construction at INFN-LNL. 


\section{References}

[1] Bao-An Li et al., Phys. Rev. C 71044604 (2005)

[2] V. Baran et al., Nucl. Phys. A 730329 (2004)

[3] F. Gulminelli et al., Eur. Phys. J. A 30 1-3 (2006)

[4] R. Hanbury, R.Q. Twiss, Nature 1781046 (1956)

[5] S. Koonin, Phys. Lett. B 7043 (1977)

[6] G. Verde et al., Eur. Phys. J A30, 81 (2006)

[7] R. Charity et al., Phys. Rev. C 523126 (1995)

[8] W. P. Tan et al., Phys. Rev. C 69061304 (2004)

[9] F. Grenier et al., Nucl. Phys. A811, 233 (2008)

[10] E. V. Pagano et al., EPJ Web of Conferences 66, 03069 (2014)

[11] G. Verde et al., Jour. of Phys: Conference Series 420(1), 012158 (2012)

[12] L. Acosta et al., Procedings IWM 2011, EPJ Web of Conferences 31, 00035 (2012)

[13] C. Boiano, et al., IEEE Trans. On Nucl. Science 511931 (2004)

[14] C.Boiano et al, devises under test.

[15] L. Acosta et al., LNS-Report , 2011-2012, pag 114

[16] L. Quattrocchi et al., EPJ Web of Conferences 66,11001(2014)

[17] P. Russotto et al., Phys. Rev. C 81, 064605 (2010)

[18] G. Grinyer et al., ACTAR TPC design report, GANIL 2013 http://pro.ganil-spiral2.eu/laboratory/detectors/actartpc/docs/actar-tpc-conceptual-design-report

[19] T. Kobayashi, T. Motobayashi, K. Yoneda, et al., SAMURAI TPC Project, MSU http://ribf.riken.jp/SAMURAI/120425_SAMURAIConstProp.pdf

[20] E.Pollacco et al., Phys. Procedia 37 1799-1804 (2012) 